\title{
AIRGLOW ROTATIONAL TEMPERATURE MEASUREMENTS DURING THE ALOHA CAMPAIGN
}

\author{
R. J. Niciejewski and J. H Yee
}

Department of Atmospheric, Oceanic, and Space Sciences, The University of Michigan

\begin{abstract}
Two instruments sensitive to nocturnal airglow emissions were flown aboard the NCAR Electra aircraft during the 1990 ALOHA campaign - a Fourier transform spectrometer and a $1 / 4$ meter grating spectrometer. The two instruments were chosen to provide complementary data in the near infrared spectral region, the former between 6000 and 10000 $\mathrm{cm}^{-1}$, and the latter between $\sim 11300$ and $\sim 11600 \mathrm{~cm}^{-1}$. Coordinated measurements were acquired by both instruments of several ro-vibrational transitions within the ground state of the Meinel band of hydroxyl and one electronic transition of the $\mathrm{O}_{2}$ At system. Rotational temperatures were deduced from both species, including several transitions within the Meinel system. It is usually assumed that the mean height of the $\mathrm{O}_{2} \mathrm{At}$ layer is several kilometers above the mean height of the $\mathrm{OH}$ layer, implying a means of determining a neutral temperature height profile, albeit coarsely. Data from two flights show an interesting behavior: temperatures inferred from $\mathrm{OH}$ and $\mathrm{O}_{2}$ At emissions from the March 31, 1990, mission are equal, $\mathrm{T}(\mathrm{OH})$ $\sim \mathrm{T}\left(\mathrm{O}_{2}\right)$, within error bars during the start of the west-to-east flight while $\mathrm{T}(\mathrm{OH})>\mathrm{T}\left(\mathrm{O}_{2}\right)$ during the latter part of the flight. For the March 22, 1990, mission, the dependences are reversed with $\mathrm{T}(\mathrm{OH})>\mathrm{T}\left(\mathrm{O}_{2}\right)$ during the first part of the southto-north flight, and temperatures similar at the end. A comparison with a standard temperature profile model for April at various latitudes indicates that the best fit is for an altitudinal temperature profile for $30 \mathrm{~N}$ latitude. A lower $\mathrm{T}\left(\mathrm{O}_{2}\right)$ indicates a high mesopause existed during the ALOHA experiment.
\end{abstract}

\section{Introduction}

Observations of airglow emissions from excited $\mathrm{OH}$ and $\mathrm{O}_{2}$ species have been used to study dynamical behavior in the terrestrial mesopause/mesosphere. Often, the literature reports studies based upon one or the other species [ Tepley, 1985; Hecht et al., 1987], and less frequently, studies based upon simultaneous measurements [Noxon, 1978]. These observations have usually been obtained from a single ground based station observing the zenith. Observations from a moving platform have been fewer [Noxon, 1964]. The airglow layers respond quickly to dynamical forcing and the response is most easily seen in the wind, density, and temperature fields associated with the emitting altitudes. Noxon, 1978, interpreted the variations in temperature from the two emitting species observed at a single site as evidence for the propagation of internal gravity waves through the mesosphere (energy upwards, phase downwards). On occasion, he observed temperature fluctuations approaching $100 \mathrm{~K}$, with some data sets indicating only a $20 \mathrm{~K}$ variance. Dual species measurements may be used to infer mesopause location.

Copyright 1991 by the American Geophysical Union.

Paper number 91GL01613

0094-8534/91/91GL-01613\$3.00
The ALOHA campaign [Gardner, this issue] was designed to measure dynamical influences in the middle atmosphere during equinox over a large water mass. The two airglow experiments flown on the NCAR Electra aircraft were chosen to provide complementary data regarding nocturnal mesospheric emissions. One instrument, the Michelson interferometer, highlighted measurements of the ground state hydroxyl Meinel transitions $\left(\mathrm{X}^{2} \Pi_{i}\right)$, in particular low upper vibrational level transitions of the $\Delta v=2$ sequence, and high upper vibrational level transitions of the $\Delta v=3$ sequence. Rotational temperatures were obtained from the (3-1) and the (8-5) transitions, though only the former will be discussed here. The grating spectrometer was primarily used to determine rotational temperatures from the electronic transition $\mathrm{O}_{2}\left(\mathrm{~b}^{1} \Sigma_{g}{ }^{+}-\mathrm{X}^{3} \Sigma_{g}{ }^{-}\right)$ At (0-1) band, though low resolution measurements of the $R$ branches of the $\mathrm{OH}$ Meinel (7-3) transition were also made.

The Michelson interferometer, an M150 model, was purchased from Bomem, Inc., in Vanier, Québec. The instrument is compact and transportable. The photodetector, an ETX $500 \mathrm{TE}$, was supplied by Epitaxx, Inc., and has an InGaAs formulation. The interferometer was mounted on a bench beneath a zenith-viewing window, the latter located immediately behind the crew cabin of the Electra. During flight operations, the light path from the window to a $45^{\circ}$ first surface mirror to the interferometer was baffled, and cabin lights in the fore section of the plane were extinguished.

During a research flight, data were usually acquired as twosided interferograms. The interferometer proved to be sensitive to gross altitude changes of the aircraft, resulting in substantial shifts of the ZPD position during ascending or descending periods and subsequent loss of data. However, when the aircraft flew at a constant altitude, the interferometer was typically stable and recorded useful data. Individual interferograms could be acquired with $\sim 5$ second temporal resolution. However, to improve the signal to noise ratio of the corresponding spectra, 30 interferograms were coadded before storage.

For the analysis reported here, several interferograms were coadded prior to transformation. The ZPD position of each of the interferograms was located and shifted for best correlation prior to coaddition. The resultant set of interferograms were then truncated with a \pm 4096 point cosine apodization function, and then zero filled before performing a $2^{15}$ point fast Fourier transform. The resultant spectra were then corrected for two effects: window transmission, and detector sensitivity, both wavelength sensitive. Window transmission was measured by observing of a weak DC white light source both with and without the zenith window. Detector sensitivity was determined by an absolute intensity calibration, using the approach described by Dandekar and Davis, 1973. Here, a tungsten filament lamp was used as a source (note that this lamp has been absolutely calibrated at NIST) illuminating a Lambertian surface. The distance from the lamp to the screen was accurately measured, and the Michelson interferometer was placed in a position where the screen overfilled the detectors field of view. This experiment was performed prior to aircraft integration of the interferometer. When performing the calibrations, the $45^{\circ}$ 
front surface mirror was removed. Wavenumber precision for all acquisitions was controlled by an internal HeNe laser, and should be accurate to $0.01 \mathrm{~cm}^{-1}$.

The grating spectrometer is fully described in Yee et al., this issue. The pointing geometry of this instrument matched the Michelson interferometer's, though it was located under a different window. The grating spectrometer was also absolutely calibrated in intensity with a similar source (same Lambertian screen) as described above.

In this short report, we will present and discuss rotational temperatures obtained by the two airglow instruments. Detailed interpretation of dynamical structure observed in the rotational temperatures and airglow intensities requires a model which accounts for aircraft motion (Doppler effect) and will not be discussed here. This analysis will be reported late in a more detailed publication.

\section{Results and discussion}

Two flights were chosen for discussion in this paper: the first was a west-east flight on March 31, 1990 (Figure 1), and the second a south-north flight on March 22, 1990 (Figure 2). The skies were clear above the aircraft on both occasions, and all aircraft experiments performed properly. Data were reduced from the Michelson interferometer experiment as described above, while grating spectrometer data were obtained as per Yee et al., this issue.

Rotational temperatures may be obtained from airglow emission data using two standard techniques based upon the resolution of the final spectrum. If individual lines within a rotation-vibration band can be recognized, then a simple technique relating individual line intensities and associated rotational term values may be used [Herzberg, 1950]. However, if the resolution of the instrument precludes the identification of rotation-vibration lines, then a least squares fit to the band profile may be performed [Tepley, 1985]. Both these techniques were used in the present analysis, the former for Michelson interferometer data and the latter for grating spectrometer data. The two experimental data sets provide an inter-

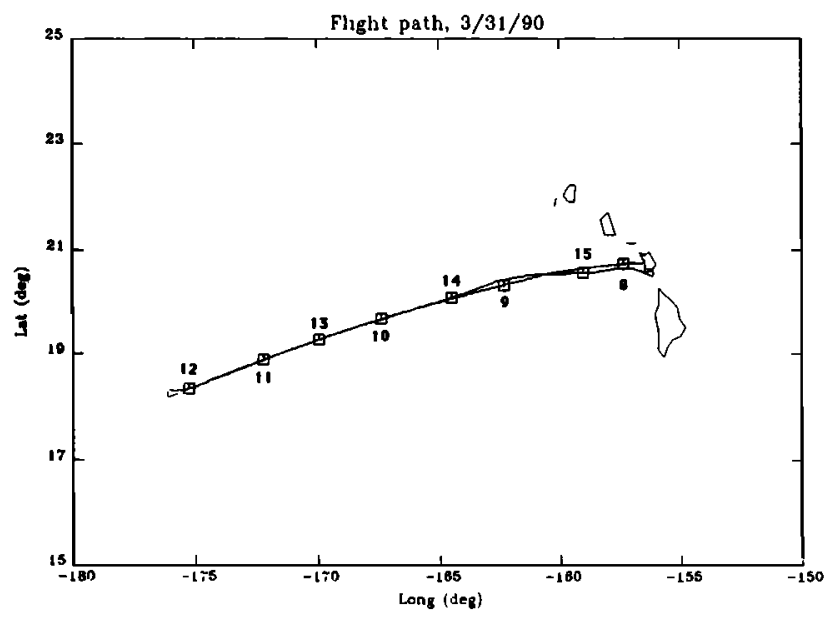

Fig. 1. Flight path for the March 31, 1990, research flight. The flight began at 07:47 UT with the aircraft headed west. Turnaround occurred at 11:50 UT at lat: $18.2 \mathrm{~N}$, long: 176.1 W. The flight ended at 15:37 UT. Boxes indicate location of the aircraft at the annotated UT hour. The Hawaiian islands are also indicated.

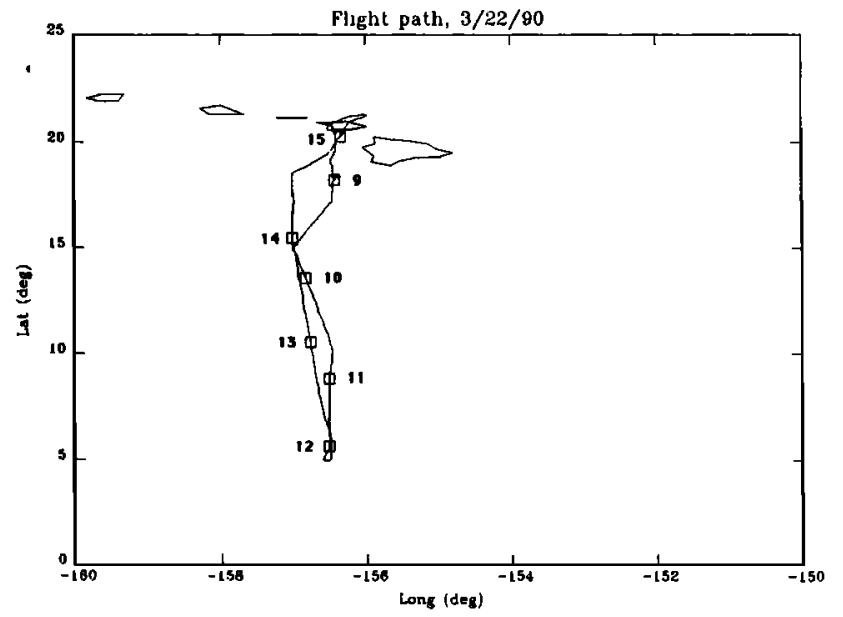

Fig. 2. Flight path for the March 22, 1990, research flight. The aircraft originated from Kahului Airport (lat: $20 \mathrm{~N}$, long: $156 \mathrm{~W})$. The flight began at 08:10 UT with the plane heading south. Turnaround occurred at 11:50 UT at lat: $4.9 \mathrm{~N}$, long: $156.6 \mathrm{~W}$. The flight ended at 15:27 UT. Note that local time $=$ UT - 10 hrs. Boxes and annotation as in Figure 1.

esting check on consistency of hydroxyl rotational temperature analysis.

Rotational temperatures and emission intensities were determined from Michelson interferometer data using a slight modification of the steps outlined by Hecht et al., 1987. Einstein coefficients for various rotational transitions were obtained from Mies, 1974. Partition function values were obtained from Krassovsky et al., 1962, while rotational term values came from Coxon, 1980, and Coxon and Foster, 1982. In the study reported here, only the $\mathrm{P}_{1}(2), \mathrm{P}_{1}(3)$ and $\mathrm{P}_{1}(4)$ lines were used in the analysis of the (3-1) OH Meinel transitions. The choice of which Einstein A factors to use for temperature analysis will lead to slightly different values for the final temperatures. We have adopted the Mies, 1974, coefficients, but note the following differences between three other recent determinations: (1) Langhoff et al., 1986; (2) Turnbull and Lowe, 1989; and (3) Nelson et al., 1990. To summarize the differences between these three determinations for the various transitions discussed in this paper, reference to tables within the citations may be used. These tables indicate that when employing (1), rotational temperatures between 3 - $6 \mathrm{~K}$ lower will be obtained compared to Mies; employing (2), temperatures between 1 - $4 \mathrm{~K}$ higher will be obtained; and employing (3), temperatures $1 \mathrm{~K}$ higher will be obtained, ie. the order would be (1) to Mies to (3) to (2), in ascending temperature order.

The derivation of rotational temperatures from the $\mathrm{O}_{2} \mathrm{At}(0$ 1) band is based upon the ability to generate accurate synthetic spectra as a function of rotational temperature. Synthetic spectra may be generated using rotational constants reported by Babcock and Herzberg, 1948. The bandpass of the grating spectrometer also transmits high rotational $\mathrm{P}$ branch lines of the OH Meinel (6-2) band as well as the $\mathrm{R}$ branches of the (73) band. Employing all the spectral information available amounts to performing a fit to the three bands, assuming identical temperatures for the two hydroxyl bands, as well as a constant background source (consisting of both sky noise and detector noise). In the initial fit, all components of the $\mathrm{OH}$ Meinel (7-3) band were used. The rotational temperatures ex- 
tracted from this run gave temperatures $\sim 30 \mathrm{~K}$ higher than corresponding temperatures obtained by the Michelson interferometer. Note that this is approximately equal to the difference cited by Nelson et al., 1990, when comparing P branch and $R$ branch temperatures using the Mies set of Einstein coefficients.

Figure 3 displays a composite plot of rotational temperatures for the March 31, 1990, flight. Shown are temperatures from the $\mathrm{O}_{2}$ At (0-1) band and the OH Meinel (3-1) band. Error bars for the oxygen band temperatures are typically $\pm 7 \mathrm{~K}$. Comparing $\mathrm{O}_{2}$ temperatures with $\mathrm{OH}$ temperatures, note that the two are similar between 08:00 UT and 11:30 UT, while the latter is warmer between 12:00 UT and 15:00 UT.

The peak altitude of emission for any airglow feature may be determined from rocket photometry through the layer. The mean altitude of peak hydroxyl volume emission rate averaged over selected rocket flights is $86.8 \pm 2.6 \mathrm{~km}$ with a thickness of $8.6 \pm 3.1 \mathrm{~km}$ [Baker and Stair, 1988]. Rocket experiments have sometimes shown an altitudinal dependence of the vibrational distribution of the ground state of hydroxyl, with low vibrational levels peaking at lower altitudes compared to high vibrational levels [Baker and Stair, 1988]. However, a recent theoretical consideration [McDade, 1991] indicates that the altitude of the peak volume emission rate should differ by no more than one to two kilometers between high and low levels. Rocket photometry has shown that the peak volume emission altitude for the $\mathrm{O}_{2}$ Atmospheric band occurs at $94 \mathrm{~km}$ [Greer et al., 1984]. Thus, there is a definite altitudinal difference between the $\mathrm{OH}$ and the $\mathrm{O}_{2}$ emitting species which may be indicated by temperature differences.

Current models of altitudinal temperature profiles [Jursa, 1985] are shown in Figure 4. Shown are results for April at several latitudes: $0,15 \mathrm{~N}$, and $30 \mathrm{~N}$. Note that in the first two cases, there is a very low mesopause and a positive temperature gradient with altitude above the mesopause. The $30 \mathrm{~N}$ case indicates a mesopause above $90 \mathrm{~km}$, though the actual temperature minimum is not indicated in the tabulation. The first part of the March 31, 1990, flight fits the latter model with an average temperature $\sim 195 \mathrm{~K}$ for the usually accepted altitude of peak hydroxyl emission. However, the second half of the flight indicates a warming in the lower hydroxyl layer perhaps signifying a dynamic effect. The higher altitude $\mathrm{O}_{2}$ temperatures remain roughly constant throughout the duration of the scientific flight suggesting that the altitude of the mesopause must lie above $90 \mathrm{~km}$ and that this region was not affected by the warming experienced at the lower altitude. A

March 31, 1990, flight

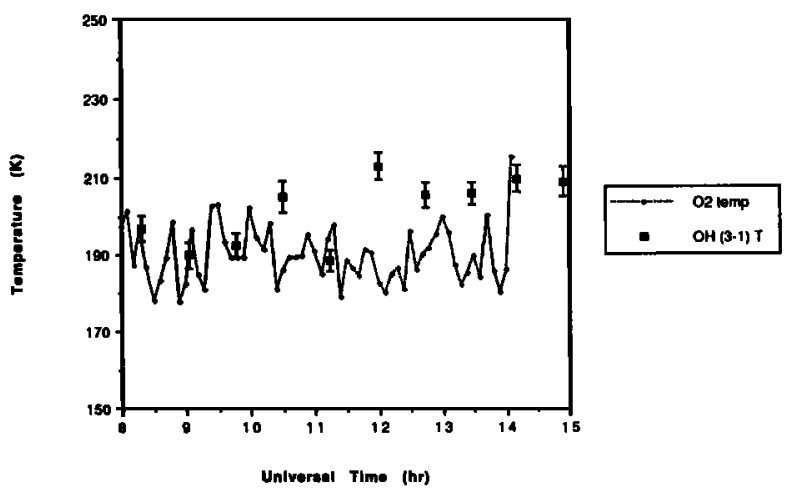

Fig. 3. Rotational temperatures observed from the NCAR Electra aircraft on March 31, 1990.

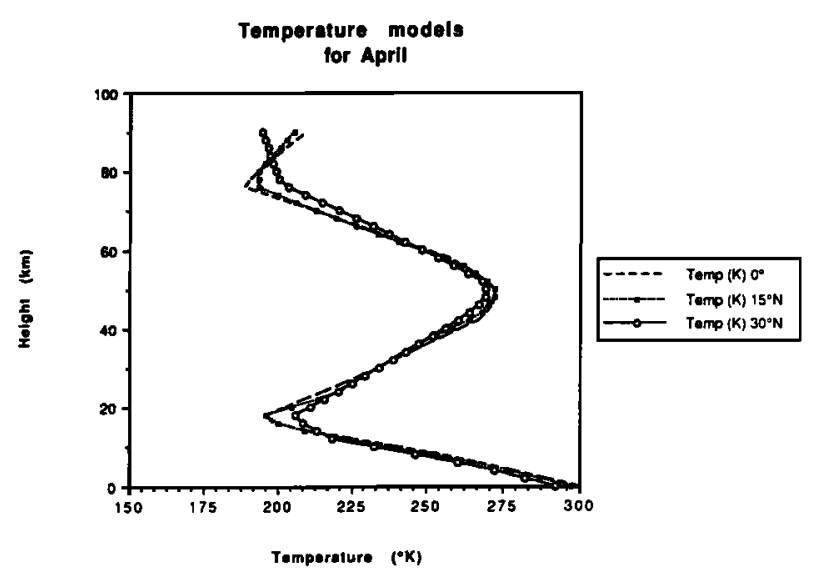

Fig. 4. Statistical temperature models from the 1985 AFGL Handbook. The models shown are valid for the equator, $15 \mathrm{~N}$, and $30 \mathrm{~N}$ for April.

similar increase in the measured hydroxyl temperature above Haleakala for the same observing period has been reported by Turnbull and Lowe, 1990. The more recent MSIS-90 model [Hedin, 1991] also displays a high mesopause $(-95 \mathrm{~km})$ in April for the equator as well as for $45 \mathrm{~N}$.

Figure 5 summarizes the aircraft airglow temperature measurements for the March 22, 1990 flight. The mesospheric conditions during this flight were most unusual and are summarized in Gardner et al., this issue. The temporal gradients of the two emissions are inverted with the $\mathrm{OH}$ temperature higher at the start of the flight than at the end, and the $\mathrm{O}_{2}$ temperature lower at lift off. Also, at the start of the flight, the temperature in the $\mathrm{OH}$ layer is greater than in the $\mathrm{O}_{2}$ layer, while at the end of the flight the two are roughly the same. The static temperature models of Figure 4 do not fit the observations very well implying that both temperatures should have peaked at the southernmost, turnaround position of the flight assuming constant altitudes for the two emitting species.

The temperature results of these two flights fall into two categories: $\mathrm{T}(\mathrm{OH}) \sim \mathrm{T}\left(\mathrm{O}_{2}\right)$ or $\mathrm{T}(\mathrm{OH})>\mathrm{T}\left(\mathrm{O}_{2}\right)$. The static AFGL models for 0 and for $15 \mathrm{~N}$ imply a substantial positive temperature gradient of roughly 16 and $10 \mathrm{~K} / 10 \mathrm{~km}$, respectively, through the $\mathrm{OH}$ and the $\mathrm{O}_{2}$ peak emission regions, while the $30 \mathrm{~N}$ model indicates a high mesopause. The recent MSIS-90 temperature profile also indicates a high mesopause

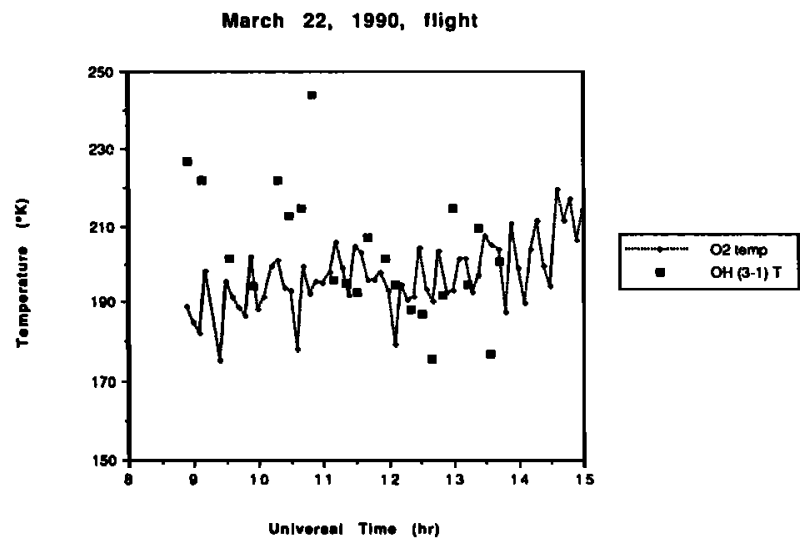

Fig. 5. Rotational temperature observed from the NCAR Electra aircraft on March 22, 1990. 
during spring equinox. Interpreting the temperature data assuming the usually accepted altitudes for peak airglow emission implies that the mesopause must be high during the campaign, and that the temperature gradient must be close to zero, or negative with increasing altitude. The observed variations in temperature as a function of position (latitude or longitude) also indicate dynamical influences in the 85 to $95 \mathrm{~km}$ range. Temporal variations between the start and the end of the two flights indicate large scale modifications to the emitting regions. In fact, the March 22 flight data were acquired during a passage under a large sporadic $\mathrm{Na}$ layer [Gardner et al., this issue]. Regarding the stability of the heights of the airglow layers, there is some evidence to indicate that there may be a latitudinal variation in height as discussed by Swenson et al., 1989. Using limb observations, they indicated that the hydroxyl airglow altitude dropped by $\sim 6 \mathrm{~km}$ over a latitudinal distance of $\sim 45$ degrees, with the $\mathrm{OH}$ airglow tangent height at the equator roughly equal to the $\mathrm{O}_{2}$ Atmospheric tangent height. It may be possible, then, that when $\mathrm{T}(\mathrm{OH}) \sim \mathrm{T}\left(\mathrm{O}_{2}\right)$ that the airglow layers have the same altitude, but without further information, it is impossible to distinguish the ambiguity between airglow layer motion and mesopause location. Additional studies of airglow derived temperatures are being conducted for the remaining flights of the ALOHA campaign, as well as data acquired during the AIDA '89 efforts.

Acknowledgements. Dr. Niciejewski was funded by NSF grant ATM-8901367. Dr. Yee was supported by NSF grant ATM-8822530. The authors would like to acknowledge Dr. I. McDade for many valuable discussions and the flight and ground crews of the NCAR Electra for their support.

\section{References}

Babcock, H. D., and L. Herzberg, Fine structure of the red system of atmospheric oxygen bands, Astrophys. J., 108, $167,1948$.

Baker, D. J., and A. T. Stair, Jr., Rocket measurements of the altitude distributions of the hydroxyl airglow, Physica Scripta, 37, 611, 1988.

Coxon, J. A., Optimum molecular constants and term values for the $\mathrm{X}^{2} \Pi(\mathrm{v} \leq 5)$ and $\mathrm{A}^{2} \Sigma^{+}(\mathrm{v} \leq 3)$ states of $\mathrm{OH}$, Can.J. Phys., 58, 933, 1980.

Coxon, J. A., and S. C. Foster, Rotational analysis of hydroxyl vibration-rotation emission bands: Molecular constants for $\mathrm{OH} \mathrm{X} \mathrm{X}^{2} \Pi, 6 \leq \mathrm{v} \leq 10$, Can. J. Phys., 60, 41, 1982.

Dandekar, B. S., and D. J. Davis, Jr., Calibrations of the airglow photometers and spectrometers, Appl. Opt., 12, 825 .

Gardner, C. S., Introduction to ALOHA-90: the airborne LIDAR and observations of the Hawaiian airglow campaign, this issue.

Gardner, C. S., T. J. Kane, J. H. Yee, R. J. Niciejewski, J. H. Hecht, R. L. Walterscheid, R. P. Lowe, and D. N. Turnbull, Formation characteristics of sporadic Na layers observed simultaneously by LIDAR and airglow instruments during ALOHA-90, this issue.

Greer, R. G. H., E. J. Llewellyn, B. H. Solheim, and G. Witt, The excitation of $\mathrm{O}_{2}\left(\mathrm{~b}^{1} \mathrm{\Sigma}_{\mathrm{g}}^{+}\right)$in the nightglow, Planet. Space Sci., 29, 383, 1981.

Hecht, J. H., R. L. Walterscheid, G. G. Sivjee, A. B. Christensen, and J. B. Pranke, Observations of wave-driven fluctuations of $\mathrm{OH}$ nightglow emission from Søndre Strømfjord, Greenland, J. Geophys. Res., 92, 6091, 1987.

Hedin, A. E., Extension of the MSIS thermosphere model into the middle and lower atmosphere, J. Geophys. Res., 96, $1159,1991$.

Herzberg, G., Molecular spectra and molecular structure. I. Spectra of diatomic molecules, Van Nostrand Reinhold Company, 1950.

Jursa, A. S. [Scientific editor], Handbook of geophysics and the space environment, Air Force Geophysics Laboratory, Chap. 14, 1985.

Krassovsky, V. I., N. N. Shefov, and V. I. Yarin, Atlas of the airglow spectrum $3000-12400 \AA$, Planet. Space Sci., 9, 883, 1962.

Langhoff, S. R., H.-J. Werner, and P. Rosmus, Theoretical transition probabilities for the $\mathrm{OH}$ Meinel system, J. Mol. Spectrosc., 118, 507, 1986.

McDade, I. C., The altitude dependence of the $\mathrm{OH}\left(\mathrm{X}^{2} \Pi\right)$ vibrational distribution in the nightglow: some model expectations, in press, J. Geophys. Res., 1991.

Mies, F., Calculated vibrational transition probabilities of $\mathrm{OH}$ (X $\left.{ }^{2} \Pi\right)$, J. Mol. Spectrosc., 53, 150, 1974.

Nelson, Jr., D. D., A. Schiffman, D. J. Nesbitt, J. J. Orlando, and J. B. Burkholder, $\mathrm{H}+\mathrm{O}_{3}$ Fourier-transform infrared emission and laser absorption studies of $\mathrm{OH}$ ( $\left.\mathrm{X}^{2} \Pi\right)$ radical: An experimental dipole moment function and stateto-state Einstein A coefficients, J. Chem. Phys., 93, 7003, 1990.

Noxon, J. F., The latitude dependence of $\mathrm{OH}$ rotational temperature in the night airglow, J. Geophys. Res., 69, 4087, 1964.

Noxon, J. F., Effect of internal gravity waves upon night airglow temperatures, Geophys. Res. Lett., 5, 25, 1978.

Swenson, G. R., S. B. Mende, and E. J. Llewellyn, Imaging observations of lower thermospheric $O\left({ }^{1} S\right)$ and $\mathrm{O}_{2}$ airglow emissions from STS 9: implications of height variations, $J$. Geophys. Res., 94, 1417, 1989.

Tepley, C. A., A nonlinear least-squares approach to the analysis of $\mathrm{O}_{2}(0-1)$ Atmospheric band emissions, Ann. Geophys., 3, 177, 1985.

Turnbull, D. N., and R. P. Lowe, New hydroxyl transition probabilities and their importance in airglow studies, Planet. Space Sci., 37, 723, 1989.

Turnbull, D. N., and R. P. Lowe, A summary of the results obtained by The University of Western Ontario groundbased Michelson interferometer during ALOHA 90, EOS Transactions, 71, 1501, 1990.

Yee, J. H., R. J. Niciejewski, and M. Z. Luo, Observations of $\mathrm{O}_{2}\left({ }^{1} \Sigma\right)$ and $\mathrm{OH}$ nightglow during the ALOHA-90 campaign, this issue.

R. J. Niciejewski, J. H. Yee, Space Physics Research Laboratory, Department of Atmospheric, Oceanic and Space Sciences, The University of Michigan, 2455 Hayward, Ann Arbor, MI 48109.
(Received April 9, 1991; revised June 7, 1991; accepted June 14, 1991.) 